\title{
A Study on Positioning Transition of R\&D Areas of Digital Camera Manufacturers
}

\author{
Jun Hosoya *
}

\begin{abstract}
In this analysis, we conducted a quantitative analysis of digital camera research and development trends, which account for $90 \%$ of global shipments by Japanese companies. Japanese companies have a mix of existing camera manufacturers and new entry electronics manufacturers. The purpose of this study was to analyze and clarify the digital camera-related patent data from the viewpoint of positioning in the $\mathrm{R} \& \mathrm{D}$ field to see how the research and development of each company's specialty technologies and technologies that need to be caught up has progressed. The research and development areas of existing camera manufacturers and new entry electronics manufacturers have been approaching since 2000 compared to the 1990s.
\end{abstract}

Keywords: Digital camera, quantitative analysis, patent data, correspondence analysis

\section{Introduction}

There is no single definition for a digital camera. Eastman Kodak invented the world's first digital camera in 1975, when the camera that converts light entering through the lens into an electrical signal by an image sensor and records and outputs it was defined as a digital camera. In connection with the invention, in May 1977, Eastman Kodak applied for a patent entitled "Electronic Still Camera (USP 4131919)". Then, in the 1980s, Japanese companies put digital cameras into practical use as analog electronic still cameras. In the 1990s, digital cameras digitized analog electrical signals from image sensors and generated and recorded images using an image processing engine. Digital cameras were expensive when they were first commercialized. Digital cameras did not form a market because of competition with video movie cameras that were introduced to the market at the same time. Under these circumstances, some digital camera developers had stopped or reduced R \& D. The digital camera "QV-10" launched by Casio Computer in 1995 contributed to the formation of the digital camera market. The QV10 achieved a low price, and a built-in LCD monitor made it possible to immediately check the captured image. In addition, the QV-10 became an explosive hit because it was able to connect to a Microsoft Windows 95-based PC released in the same year. With the launch of the digital camera market along with the QV-10 hit, existing camera manufacturers, photographic film manufacturers, and home appliance manufacturers entered the market at once. Although the

\footnotetext{
* Waseda University, Tokyo, Japan
} 
digital camera market was expanding, many companies had withdrawn due to intense market competition. Meanwhile, due to the market competition, the development of digital camera product technology had been progressing rapidly. According to CIPA (Camera \& Imaging Products Association in Japan), shipments of this digital camera increased from 5.09 million in 1999 to 121.46 million in 2012. In this process, the number of film cameras shipped exceeded the number of film cameras shipped in 2002, and the leading role in the camera market was replaced. Japanese companies have a high share of digital cameras. All five top-selling companies in the global market in 2018 are Japanese companies, with a market share of over $85 \%$. In this way, what kind of digital camera brand manufacturers is the market rapidly rising and Japanese companies have strength in? These manufacturers can be classified into the following two types. The first category includes companies that have been camera manufacturers since the film camera era. In this paper, these companies are called "existing camera manufacturers". The second category includes companies that are new entrants into the market from digital cameras. In this paper, these companies are referred to as "new entry electronics manufacturers". The reason why digital camera manufacturers can be classified in this way is as follows. This is because the digital camera is a fusion product of the technology used continuously from the old fashioned film camera and the digital technology accompanying the development of IT. It is thought that existing camera manufacturers are good at technologies such as lens mechanisms and shutters that are used continuously from old fashioned film cameras. On the other hand, it is considered that the new entrant electronics manufacturers are good at technologies such as image processing and image elements, which become digital technologies with the progress of IT. In other words, Japanese companies that handle digital cameras have their own technical fields. On the other hand, because it is a fusion product, it seems that existing camera manufacturers who have been engaged in cameras since the old silver halide film era need to catch up with digital technology as IT advances. Similarly, new entry electronics manufacturers who are good at digital home appliances will need to catch up with the technology that will continue to be used from old fashioned film cameras. In other words, existing camera manufacturers and new entry electronics manufacturers need to catch up with each other's expertise. In this analysis, more than $80 \%$ of the world's shipments are represented by Japanese companies that have a mix of existing camera manufacturers and new entry electronics manufacturers. It analyzes whether it has evolved and accumulated. I analyzed digital camerarelated registered patent data in terms of accumulating R \& D results. I analyzed by clarifying the positioning of each manufacturer using patent data held in chronological order.

\section{Preceding Studies}

In economic growth theory, technological progress (increased productivity) is the source of growth [1]. In order to evolve the technological progress, companies carry out R \& D. R \& D is also described as combining production factors to enable the acquisition of new knowledge [2]. For example, R \& D expenditure per worker has been shown to have a positive effect on corporate growth against the growth rate of Japanese manufacturing [3]. The following past studies have stated that corporate research activities affect product superiority and, as a result, generate profits. Companies carry out research and development as one measure to maintain competitive advantage. Management resources owned by companies are the source of competitive advantage. Many researchers have argued that competitive advantage will be built as a result of utilizing their management resources [4] [5] [6]. Strategic studies have argued that 
the resources held by individual companies are heterogeneous and that they are the source of competitive advantage [4]. The research approach looks inside companies and points out that each company has different management resources and capabilities, and that heterogeneity leads to differentiating products and services. Some have argued that companies focus on their core management resources and that, due to their heterogeneity, companies can gain a competitive advantage [7]. The patents measures reflecting the volume of companies' research activity were reliably associated with the future performance of R\&D-intensive companies in capital markets [8]. Cooper (1987) concluded that product superiority is the quantity one factor influencing commercial success and that project definition and early, predevelopment activities are the most critical steps in the new products development process [9]. Henard (2004) stated that there is a strong correlation between new product advantage and new product performance [10]. Song (1997) stated that it can achieve high performance in the market, such as sales and profits, when differentiated from competing products [11]. Improving the performance of a new product was an important result of the superiority of the new product [12]. In qualitative and quantitative studies on the result analysis of R\&D in companies, it is generally accepted to approach it by analyzing patent and patent applications data [13]. This is predicated on the idea that it is greatly useful because patent data covers all technical fields by time-sequentially. Therefore, especially in large companies, patent date is used as one of the predominant indexes for research and development results [14]. Patents are one of the R \& D outputs. And the activities related to patents of companies are closely related to $\mathrm{R} \& \mathrm{D}$ expenses. It is further stated that patents are a good indicator of differences in invention activity between different companies [15] [16]. As for R\&D activities and patent data in firms, it has been reported that in almost all industries in Japan, the more research and development budget there is, the more the quantity of patent applications increases. In addition, credibility of evaluation of $R \& D$ activities using patent date and its results has been established [17]. Also, Kendall (2010) discovered correlations between R\&D investment costs and the quantity of patents and between the quantity of patents and new products after analyzing R\&D investment costs, the quantity of patents and new products from over 19year samples from 35 industries and 272 companies [18]. Thus, analysis on the quantity of patent applications by companies is an important indicator not only of quality and effectiveness of R\&D from the perspective of its correlation with R\&D expenses, but also it is used to predict the future possibilities of $R \& D$ strategies as the outcome of $R \& D$ expenses, as well. Moreover, there are some unique studies, which aim at determining not the effectiveness or direction of a specific company's R\&D, but the trend of a specific technical field, and progress or direction of R\&D by companies in the field. In Pilkington and Dyerson's study (2006), they examined patent applications regarding the electronic automobile field and investigate content and the quantity of patent applications by key R\&D players (companies) in the field [19]. As a result, they conclude that the speed of R\&D in the electronic automobile field was affected and accelerated by exhaust regulations for environmental countermeasures. Furthermore, it was stated that in order to increase market value, it is necessary to invest more resources in research and development activities and to enhance the advantages in the most important technical fields [20]. It is noted in several papers that patent management is important for marketing. The role of marketing in patent management is explained, including technology, positioning and licensing [21]. In addition to these viewpoints, there are also papers that add the viewpoints of existing companies and new entrants as follows Lieberman surveyed the theoretical and empirical literature on mechanisms that confer advantages and disadvantages on first-mover firms [22]. It describes incentives for new companies and new entrants to invest in markets where innovation has occurred. It also states that existing companies have lower productivity than newcomers [23]. 
Like the former, it has been analyzed that it is important to make effective use of the company's existing resources even when entering a new market [24]. Schnars analyzed the quantification and qualitative analysis of how new entrants retain a dominant position in the market [25]. Existing companies can develop existing technologies most quickly, and are skilled at developing new products by combining new technologies and existing technologies through the acquisition and accumulation of new technologies and resources [26]. This paper focuses on digital camera patents. In addition, we analyze digital camera-related companies by classifying them into existing camera manufacturers and newly entered electronics manufacturers. When the market for digital cameras began to rise, it was analyzed that the industries involved belong to the way companies view digital cameras [27]. It is analyzed in past papers that it is important for companies to diversify like the new entrants of digital camera manufacturers described in this paper [28] [29].

Next, I will describe the previous research on technology fusion. Kodama (1992) uses the term "technology fusion" to fuse different types of technology when explaining the characteristics of innovation that Japanese companies are starting from [30]. Kodama cites mechatronics (NC machine tools) and optronics as specific examples of this "technology fusion." The reason for using the term fusion is that, for example, two types of technology will be combined into one or more technologies. The digital camera used in this paper is a combination of old fashioned silver halide film camera and digital technology. It is considered that "technology fusion" has occurred because the market size of digital cameras has expanded dramatically. Suzuki $(2004,2007)$, who quantitatively analyzes this concept of "technology fusion", uses the concept of Co-occurrence of IPC (International Patent Classification), which is a technology classification of patents. What we do is called "technology fusion," and we analyze the fusion process in time series of the number of patent applications [31] [32]. This concept is inherited and developed, and "Technology fusion degree analysis" is also implemented in this paper.

\section{Hypothesis Setting}

As mentioned above, the digital camera is a fusion product of the technology continuously used from the old film camera and the rapidly developing digital technology. In 2000, a market was set up that shipped more digital cameras than old fashioned film cameras. The top manufacturers in the market are a mixture of existing camera manufacturers and newly entered electronics manufacturers. Therefore, the research and development approach to this market should be different. On the other hand, the market has risen since the digital camera QV-10 was released by Casio in 1995. Over the next three years, 30 or more digital camera makers entered the market. This was because it was easy to enter new fields immediately after the rise of the digital camera market. This was because the digital camera of this period had a short road to commercialization due to modularization. If the external interfaces of the parts that were realized by modularization were unified, cooperative operation between the parts would not be necessary, and each technical element will be emphasized. In addition, the performance of digital technology such as image elements and image processing of digital cameras around this time was inferior to that of film technology, which is an analog technology equivalent to that. Therefore, the technology required for digital cameras was not higher than that of fill cameras at the time. However, this situation will change completely after 2000 . When the Casio QV-10 appeared, the number of pixels that showed the fineness of the captured image was about 250,000, but it has exceeded 1 million and 
has increased further. In 2002, digital camera shipments were higher than analog camera shipments. In order to increase the number of pixels and bring out their performance, not only digital technology of digital cameras but also analog technology such as optical technology is required to have a high level of performance. In addition to the fact that the high degree of integration between analog and digital technologies not only creates a barrier to entry into the digital camera field, but also contributes to the competitiveness between manufacturers, companies that cannot make high-level connections will withdraw. Based on these, in this paper we decided to evaluate the following ideas quantitatively. In the 1900s, existing camera manufacturers should have been conducting research and development focusing on analog technology in the old silver salt film era. In the 2000s, along with the need to differentiate from other companies and the sophistication of performance required for digital cameras, we moved to research and development in the field of integration of digital analog technology and digital technology, and further integration. The degree should have increased. After that, even if the product called a compact digital camera, which is technically simple among digital cameras, can no longer be differentiated, the single-lens reflex camera, which is a technically high-level system camera, has Connections have become even higher, and have become a high barrier to entry into this field. In summary, when the market for digital cameras was rising, digital camera makers were able to create products by combining modules. Since then, with the progress of digital camera technology, a fusion technology of analog technology and digital technology has become necessary. On the other hand, digital camera makers include existing camera makers who are good at analog technology and new entrant electronics makers who are good at digital technology. Therefore, these manufacturers with different positions should have made progress in their research and development.

Therefore, in this paper, Hypothesis 1 was set regarding fusion in research and development.

Hypothesis 1.

Since 2000, existing camera manufacturers have moved from analog technology to integrated research and development of analog and digital technology.

In addition, after 2000, new entrants to electronics manufacturers moved from research and development centered on digital technology to research and development in the field of integrated technology with analog technology, and the degree of integration seems to have increased. On the other hand, Hypothesis 2 was set in this paper.

Hypothesis 2.

Since 2000, new entrants to electronics manufacturers have moved from digital technology to integrated research and development of analog and digital technology.

In the single-lens reflex market, which is a system camera, technical competition among manufacturers is still continuing. However, considering the impact of compact digital cameras, where differences can no longer be found in the market, I think the differences in research and development of digital cameras as a whole will be smaller. With the progress of research and development, existing camera makers who are good at analog technology and new entrants that are good at digital technology are expected to resolve the differences in their R\&D areas. Therefore, we set the third hypothesis.

Hypothesis 3.

Since 2005, when commoditization becomes remarkable, the R\&D areas of existing camera makers and new entrants are close to each other. 
We will verify these three hypotheses in the following sections.

\section{Methodology}

\subsection{Analyzed company}

In this analysis, we divide Japanese digital camera manufacturers into existing camera manufacturers and new entry electronics manufacturers. The purpose of this study is to analyze and clarify camera-related patent data on how each group's specialty technologies and the R \& $\mathrm{D}$ results of technologies that need to be caught up are reflected in the market. The target companies are the world's top digital camera shipment companies, targeting existing camera manufacturers from the film age (Canon, Nikon, Fuji film, Olympus) and new entry electronics manufacturers from the digital camera age (Sony, Panasonic, Casio).

\subsection{Target data}

In this analysis, patent data is used as the product of research and development. To identify which technology the patent is, use the classification symbol called IPC (International Patent Classification), which is attached to the patent application publication/registration data. IPC (International patent code) is used to specify the kind of technology of patents. IPC is the worldwide technological classification code. The Patent Office gives IPC to patent data. A specialized examination official of the technical field of the Patent Office concerned gives IPC to these for application data. Thus, it is thought that the reliability of the given sequence is high. More than one of IPC are usually given to each patent data. IPC which symbolizes technology of the invention data applied for is called "Main IPC". "Main IPC" is given certainly to all bibliographies. More than one technology are sometimes included in one of invention. The Patent Office gives more than one IPC to the invention in that case. All except for Main IPC is called CO - IPC in more than one IPC. This IPC is used to specify a digital camera related patent by this analysis. "The patent which makes the analog part from the film age the main feature" is called "analog patent" by this analysis. "The patent which makes the technology added newly from a digital camera the main feature" is called "digital patent". IPC is used for difference between this "analog patent" and "digital patent". Since putting it in the analysis target, a fusion patent of "analog patent" and "digital patent" is also considered. It's judged by Main IPC in which field it's a characteristic patent. When Main IPC is "technological classification of the lens and optical subsystem: G02B1 - G02B17" or "technological classification of a camera: G03B1 G03B19" the invention is called "analog patent". When Main IPC is "technological classification related to digital processing: H04N5/22 - 25" or "technological classification of image pickup device: H04N5/33 - 36, H01L27/146 - 148", the invention is called "digital patent". These technological classifying processes were put into effect by making reference to a document (Japan Patent Office technical report). Data for analyses uses applied patents for Japan Patent Office. From the patents applied to the Japan Patent Office, those that are analog and digital patents were extracted from 1995 to 2014 on the basis of filing date. The applied patent will be published at least 1 year and 6 months after the application is filed, unless a request for accelerated examination is made. The date of searching the patent data prepared for this analysis was conducted in April 2019, and does not affect the analysis of patent data filed by 2014. As mentioned above, analog and digital patents are identified by the "main IPC", but in this analysis, they are further classified into three categories. Some patents have only one IPC, while others have multiple IPCs. Multiple IPCs granted in one patent may be technically similar fields or 
different technical fields. In other words, when multiple IPCs are assigned, even if the patent is judged to be an analog patent by the main technology, it cannot be concluded that the patent does not include technology in any other field. This is because the "main IPC" may be an analog patent and the "CO-IPC" may be other than an analog patent. Therefore, for each of the analog and digital patents, the degree of technology fusion is classified into three levels by utilizing the technology classification of patents. The higher the degree of technology fusion, the more patents include technologies in different fields. The IPC assigned to each patent is used to measure the degree of technological fusion. IPC is a classification symbol with a hierarchical structure. The hierarchy is composed of sections, classes, subclasses, main groups, and subgroups from the top, and the technical fields are subdivided toward the bottom. The IPCs that are the same at the main group level are technically closer to each other than the same IPCs at the section level because they are the same technology in a hierarchy where the technical fields are subdivided. In other words, a patent with a different IPC at the section level, which is the highest level, contains the technology in the most different field. By utilizing this hierarchical structure, we grasp the level at which the technical fields of each patent differ and measure the degree of technological fusion in three levels. Patents with a low degree of technological fusion in this analysis are classified into the following two patterns. "Patents with only the main IPC" or "Patent that causes a difference in IPC in the subgroup". Patents with a medium degree of technological fusion in this analysis are classified into the following two patterns. "Patent that causes a difference in IPC in the main group" or "Patent that causes a difference in IPC in the subclass". Patents with a high degree of technological fusion in this analysis are classified into the following two patterns. "Patents that causes a difference in IPC in the class" or "Patent that causes a difference in IPC in the section". For example, assume that there is a patent in which the main IPC is G02B1/00 and the CO-IPC is G02B3/00. In this case, the difference between the two IPCs occurs at the Main Group level (the same up to the Sub Class level of G02B). Therefore, the degree of technological fusion is judged to be Medium.

Table 1: IPC hierarchy (example: G02B3/02)

\begin{tabular}{lll}
\hline \multicolumn{1}{c}{ IPC level } & \multicolumn{1}{c}{ Explanation } & \multicolumn{1}{c}{ example:G02B3/02 } \\
\hline Section & First one character & G \\
Class & First three characters & G02 \\
Subclass & First four characters & G02B \\
Main group & Character before slash & G02B3 \\
Sub group & All characters & G02B3/02 \\
\hline
\end{tabular}

Table 2: The degree of technological fusion

\begin{tabular}{ll}
\hline \multicolumn{1}{c}{$\begin{array}{c}\text { The degree } \\
\text { of technological fusion }\end{array}$} & \multicolumn{1}{c}{ The level at which the IPC difference occurs. } \\
\hline $\begin{array}{l}\text { High } \\
\text { Medium }\end{array}$ & $\begin{array}{l}\text { Section level, Class level } \\
\text { Subclass level, Main group level } \\
\text { Low }\end{array}$ \\
\hline
\end{tabular}

The effects obtained by measuring the degree of technological integration are described below. According to the "Survey on Research Activities of Private Enterprises" by the Ministry of Internal Affairs and Communications of Japan, research and development is divided into basic research, applied research, and development research. Basic research is defined as "theoretical or experimental research conducted to form new hypotheses or theories or to obtain new knowledge 
about phenomena or observable facts without directly considering special applications or uses". Applied research is defined as "searching for new applied methods, using the knowledge discovered in basic research, to set specific goals and confirm the feasibility of practical application, and for methods already in practical use. Research". Similarly, developmental research is defined as "use of knowledge gained from basic research, applied research, and actual experience, with the aim of introducing new materials, devices, products, systems, processes, or improving existing ones. R\&D”. In this way, it is considered that many technological elements are combined in development research that emphasizes social implementation rather than basic research and applied research that emphasize knowledge discovery. As mentioned above, patents are assigned one or more hierarchical technology classifications called IPCs. By measuring the degree of technology fusion based on the IPCs assigned to each company at regular intervals, it becomes possible to understand where the research stage emphasizes basic, applied, and development. In addition, by using the main IPC that represents the main technical features, it becomes possible to understand which technology is emphasized in the original technology of the fusion technology product fusion. By using the degree of fusion of technologies in different fields, it is possible to determine whether an analog patent is a patent specialized in analog technology or a patent combined with technologies in different fields.

\subsection{Analysis method.}

In this analysis, correspondence analysis is used to grasp the transition of the positioning of digital camera manufacturers in the research and development area. The numerical values used for the correspondence analysis are the totals of the digital camera related patents applied to the Japan Patent Office from 1990 to 2014 by each of the seven digital camera manufacturers targeted for the analysis. The row and column items of the cross tabulation table used for correspondence analysis are as follows. The line items are the five periods every five years from 1990 to 2014 for each of the seven digital camera manufacturers targeted in the analysis. The column items are classified into analog patents or digital patents for each patent application and further classified into three categories according to the degree of technological fusion. In the correspondence analysis, both the rows and the columns are rearranged so that the correlation coefficient between the row elements and the column elements is maximized, using the cross tabulation table as the original data. After that, in the correspondence analysis, the elements of the rows and the elements of the columns are expressed in a two-dimensional space. It is possible to visually grasp the similarity between multiple categories and the depth of the relationship from the plotted distances.

\section{The Outline of Analysis Data}

In this analysis, the number of patent applications related to digital cameras from 1990 to 2014 is used. In analog patents, the number of applications by existing camera manufacturers has exceeded the number of applications by new entry electronics manufacturers in each period. Canon has ranked first in the number of applications for the entire period. All three new entry electronics manufacturers have the largest number of applications in the period from 2005 to 2009. 


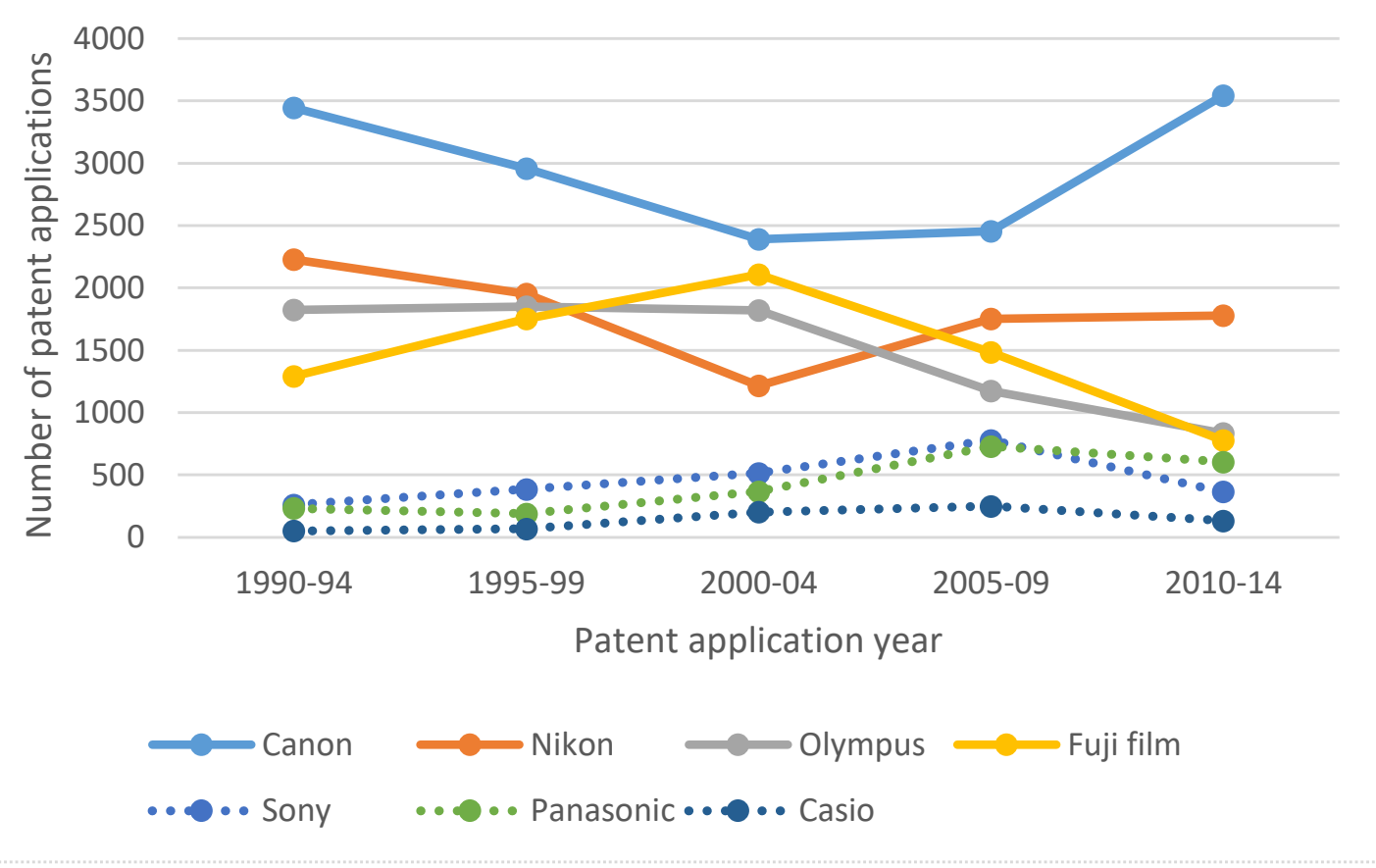

Figure 1: Number of patent applications every 5 years (analog patent)

Except for Canon, the number of digital patent applications peaks in the period from 2005 to 2009. Only Canon has the largest number of applications from 2010 to 2014. Unlike analog patents, new entry electronics manufacturers are always ranked in TOP3. On the other hand, digital patents, like analog patents, have more applications for Canon than other companies, and are always in the top two positions.

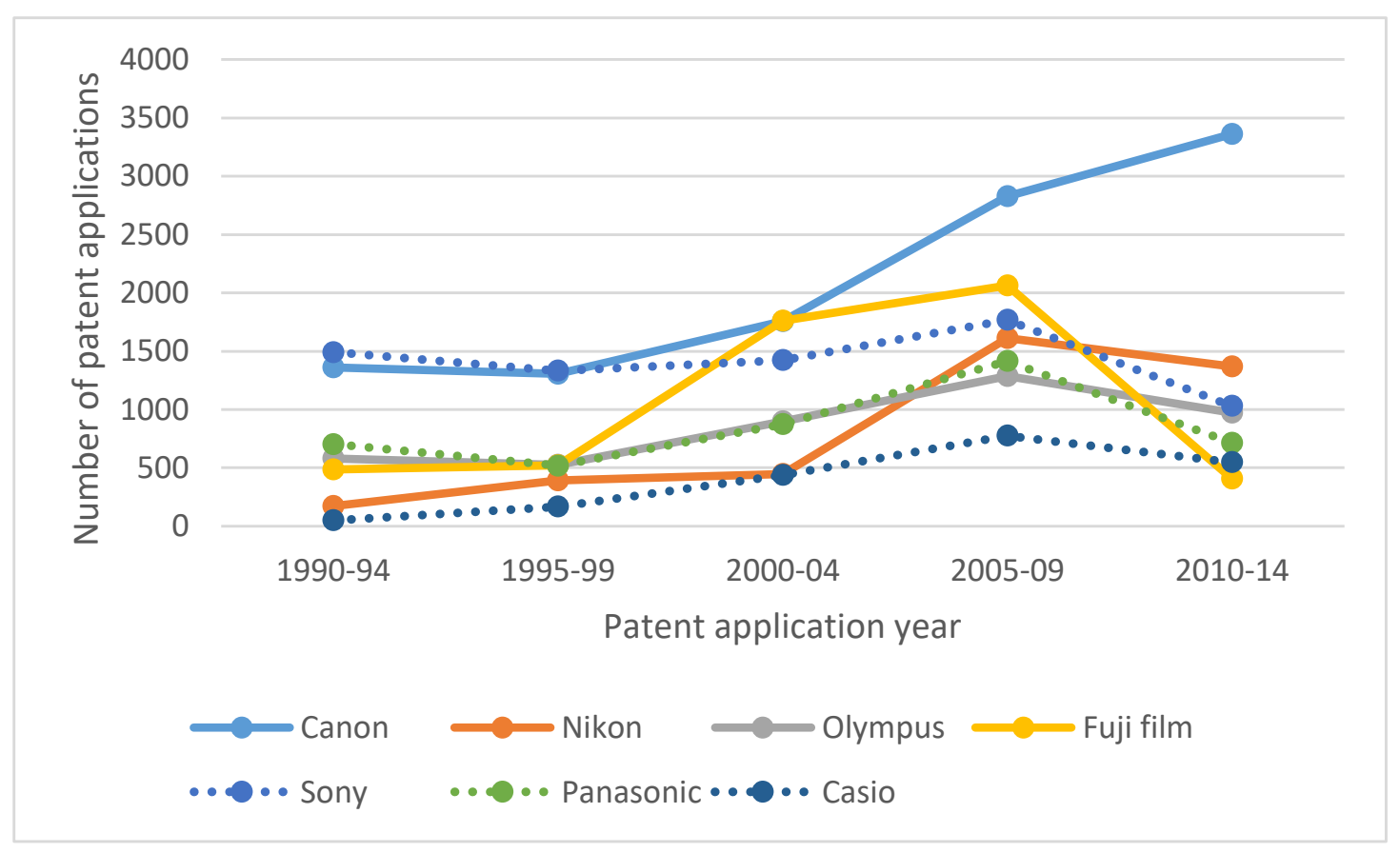

Figure 2: Number of patent applications every 5 years (digital patent) 


\section{The Result of Analysis}

Correspondence analysis was performed according to the analysis method in Chapter 4 . Of the 5 dimensions extracted by correspondence analysis, the contributions of the inertias of dimension 1 and dimension 2 were $70.3 \%$ and $18.7 \%$, respectively, and the two dimensions explained $89.0 \%$ of the total data. The singular values of dimension 1 and dimension 2 were 0.409 and 0.211 , respectively. The figure 3 below shows a plot of dimension 1 and dimension 2 . Table 3 shows the abbreviations for each company in Figure 3. Dimension 1 can be interpreted as showing digital patents in the positive direction and analog patents in the negative direction. Dimension 2 can be interpreted as a low degree of technological fusion in the positive direction and a high degree of technological fusion in the negative direction. Furthermore, cluster analysis was performed based on the coordinates of dimension 1 and dimension 2 obtained by correspondence analysis, and they were classified into 4 clusters (Figure 4). The four clusters were named clusters 1 to 4 . In the clustering method, Euclidean distance was used for distance calculation. Similarly, the distance calculation after the merger used the Ward method. Cluster 1 includes those with a low degree of analog technology fusion (notation: A_Low) and medium (notation: A_Medium). Cluster 1 includes all existing camera manufacturers in the 1990s (1990 to 1994,1995 to 1999). On the other hand, existing camera manufacturers since 2000 and new entry electronics manufacturers are not included. Cluster 2 includes those with a high degree of analog technology fusion (notation: A_High). And Cluster 2 includes all 2000s of existing camera manufacturers (2000 to 2004, 2005 to 2009, and 2010 to 2014). It also includes Panasonic 2005 and Casio 1990. Cluster 3 includes those with a high degree of digital technology integration (notation: D_High) and medium levels (notation: D_Medium). And cluster 3 includes many new entry electronics manufacturers from the 2000s. On the other hand, existing camera manufacturers are not included. Cluster 4 includes those with a low degree of digital technology integration (notation: D_Low). And Cluster 4 includes all of the new entry electronics manufacturers Sony and Panasonic from the 1990s. On the other hand, Casio, a new entry electronics manufacturer, and those of existing camera manufacturers are not included. 


\section{Dimenşion $1 \times$ Dimension 2}

- Analog / The degree of technological fusion

- Digital / The degree of technological fusion

$\triangle$ Existing camera manufacturers 1900s 1.5

Existing camera manufacturers 2000s

* New entry electronics manufacturers 1900 s

- New entry electronics manufacturers 2000s

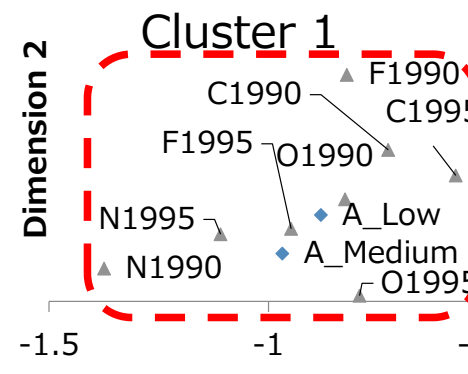

0.5
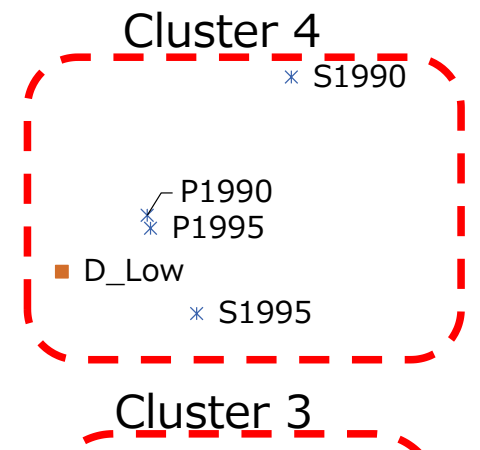

Cluster

$*$ Ca1995

Cluster 2

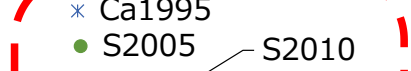

- P2005

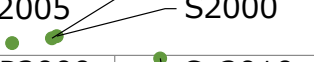

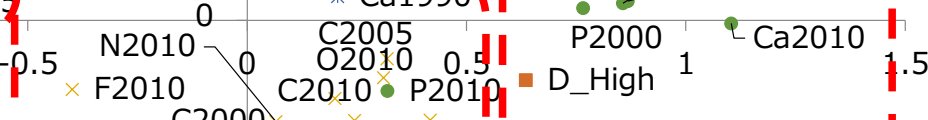

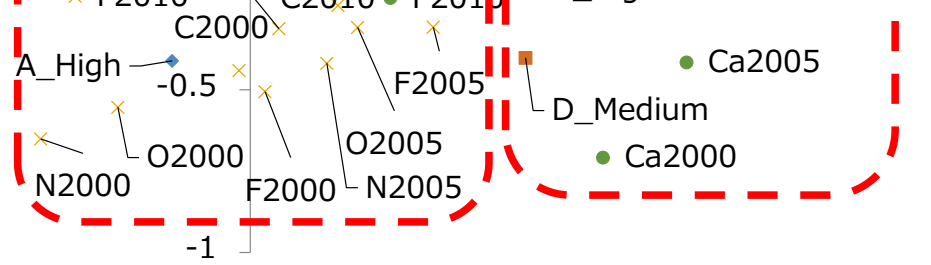

Dimension 1

Figure 3: Correspondence analysis results

Table 3: Abbreviation of each company in Figure 3

\begin{tabular}{llllllll}
\hline $\begin{array}{l}\text { Application } \\
\text { period }\end{array}$ & Canon & Nikon & $\begin{array}{c}\text { Fuji } \\
\text { film }\end{array}$ & Olympus & Sony & Panasonic & Casio \\
\hline $\begin{array}{l}\text { From 1990 } \\
\text { to } 1994\end{array}$ & $\mathrm{C} 1990$ & $\mathrm{~N} 1990$ & $\mathrm{~F} 1990$ & $\mathrm{O} 1990$ & $\mathrm{~S} 1990$ & $\mathrm{P} 1990$ & $\mathrm{Ca} 1990$ \\
$\begin{array}{l}\text { From 1995 } \\
\text { to 1999 }\end{array}$ & $\mathrm{C} 1995$ & $\mathrm{~N} 1995$ & $\mathrm{~F} 1995$ & $\mathrm{O} 1995$ & $\mathrm{~S} 1995$ & $\mathrm{P} 1995$ & $\mathrm{Ca} 1995$ \\
$\begin{array}{l}\text { From 2000 } \\
\text { to 2004 }\end{array}$ & $\mathrm{C} 2000$ & $\mathrm{~N} 2000$ & $\mathrm{~F} 2000$ & $\mathrm{O} 2000$ & $\mathrm{~S} 2000$ & $\mathrm{P} 2000$ & $\mathrm{Ca} 2000$ \\
$\begin{array}{l}\text { From 2005 } \\
\text { to 2009 }\end{array}$ & $\mathrm{C} 2005$ & $\mathrm{~N} 2005$ & $\mathrm{~F} 2005$ & $\mathrm{O} 2005$ & $\mathrm{~S} 2005$ & $\mathrm{P} 2005$ & $\mathrm{Ca} 2005$ \\
$\begin{array}{l}\text { From 2010 } \\
\text { to 2014 }\end{array}$ & $\mathrm{C} 2010$ & $\mathrm{~N} 2010$ & $\mathrm{~F} 2010$ & $\mathrm{O} 2010$ & $\mathrm{~S} 2010$ & $\mathrm{P} 2010$ & $\mathrm{Ca} 2010$ \\
\hline
\end{tabular}




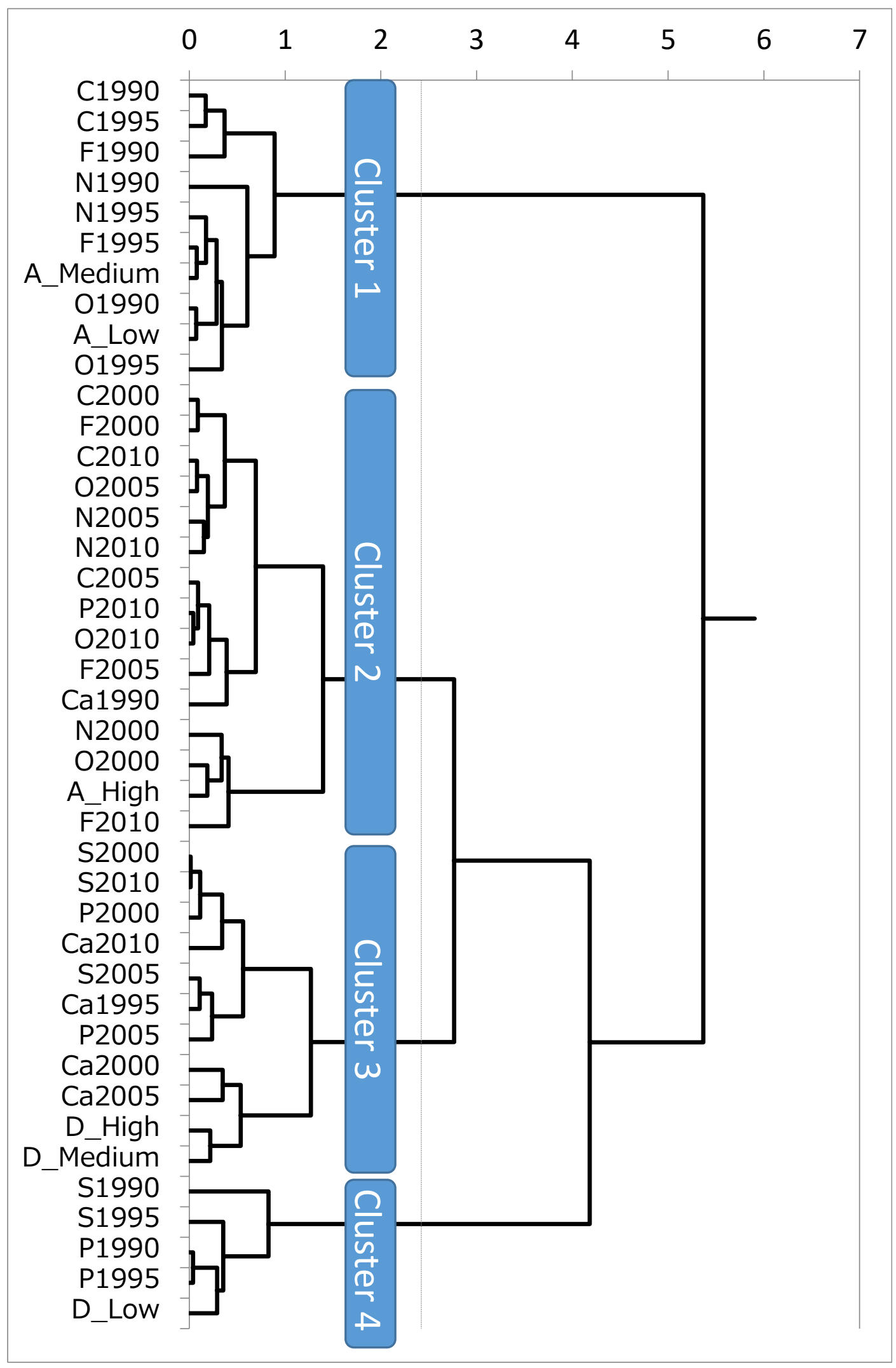

Figure 4: Correspondence analysis results 
Table 4: Manufacturers by age included in each cluster in Figure 3

\begin{tabular}{lcccc}
\hline Cluster No. & $\begin{array}{c}\text { 1990s } \\
\text { existing camera } \\
\text { manufacturers }\end{array}$ & $\begin{array}{c}\text { 1990s } \\
\text { new entry } \\
\text { electronics } \\
\text { manufacturers }\end{array}$ & $\begin{array}{c}\text { 2000s } \\
\text { existing camera } \\
\text { manufacturers }\end{array}$ & $\begin{array}{c}\text { 2000s } \\
\text { new entry } \\
\text { electronics } \\
\text { manufacturers }\end{array}$ \\
\hline Cluster 1 & $\begin{array}{c}\text { C1990 N1990 } \\
\text { F1990 O1990 }\end{array}$ & & & \\
& C1995 N1995 & & & \\
Cluster 2 & F1995 O1995 & Ca1990 & C2000 N2000 & P2010 \\
& & & F2000 O2000 & \\
& & & C2005 N2005 & \\
& & & F2010 O2005 2010 & \\
Cluster 3 & & Ca1995 & & S2000 P2000 \\
& & & Ca2000 \\
& & & Ca2005 P2005 \\
& & & & S2010 Ca2010 \\
Cluster 4 & & & & \\
& & S1990 P1990 & & \\
\hline
\end{tabular}

\section{Hypothesis Verification and Consideration}

\subsection{Hypothesis verification}

\subsection{Verification of Hypothesis 1}

The hypothesis set in Chapter 3 is verified. Hypothesis 1 is "In the 1990s, the existing camera manufacturers moved from analog technology to integrated research and development of analog and digital technology". In Figure 4, the existing camera manufacturers in 1990 are all included in cluster 1 . This cluster 1 includes low or medium degree of analog technology fusion. In other words, analog technology research and development can be said to be a feature of clusters 1 . The existing camera manufacturers of the 2000s are included in cluster 2 . This cluster 2 includes those with a high degree of analog technology fusion. In other words, it can be said that Cluster 2 is characterized by the fact that research and development that integrates analog technology and other technologies is the main focus. In addition, this cluster 2 is adjacent to the cluster 3 that includes those with a high degree of digital technology integration. In other words, it can be said that Cluster 2 is conducting research and development closer to digital technology than Cluster 1. This supports the conclusion of Hypothesis 1.

\subsubsection{Verification of Hypothesis 2}

Hypothesis 2 is "In the 1990s, new entry electronics manufacturers moved from digital technology to integrated research and development of analog and digital technology". In Figure 
4, Sony and Panasonic in the 1990s are all included in cluster 4. This cluster 4 includes those with a low degree of digital technology fusion. In other words, it can be said that R\&D centered on digital technology is a feature of cluster 4. And most of the new entrants in the 2000s (excluding Panasonic from 2010 to 2014) are included in cluster 3. This cluster 3 includes those with medium or high degree of digital technology integration. In other words, it can be said that the characteristic of cluster 3 is that the main focus is on integrated research and development of digital technology and other technologies. In addition, this cluster 3 is adjacent to the cluster 2 that contains the ones with a high degree of analog fusion. In other words, it can be said that cluster 3 is conducting research and development closer to de-analog technology compared to cluster 4. However, Casio, a new entry electronics manufacturer, is included in cluster 2 from 1990 to 1994 and in cluster 3 after 1995. Considering these points, Sony and Panasonic among the new entry electronics manufacturers support the establishment of Hypothesis 2. However, since Casio has been mainly engaged in integrated research and development of analog and digital technologies since the 1990s, the establishment of Hypothesis 2 is not supported. Therefore, in this analysis, the establishment of Hypothesis 2 is not supported by all new entry electronics manufacturers.

\subsubsection{Verification of Hypothesis 3}

Hypothesis 3 is "after 2005, when commoditization becomes remarkable, the R\&D areas of existing camera manufacturers and new entry electronics manufacturers are close". In Fig. 4, existing camera manufacturers are included in cluster 2 only after 2005. Similarly, all new entry electronics manufacturers are included in Cluster 3 except for Panasonic from 2010 to 2014. In the 1990s, existing camera manufacturers were included in cluster 1 only, and new entry electronics manufacturers Sony and Panasonic were included in cluster 4. It can be said that the regions of clusters 2 and 3 are closer than the regions of clusters 1 and 4 . However, even after the 2000 s, it seems that the research areas are not close enough to the extent that existing camera manufacturers and new entry electronics manufacturers coexist in the same area. Therefore, in this analysis, the establishment of Hypothesis 3 is not supported.

\subsection{Consideration}

\subsection{Consideration of Hypothesis 1 and Hypothesis 2}

Of the three hypotheses set in Chapter 3, only hypothesis 1 supported the conclusion. The difference between Hypothesis 1 and Hypothesis 2 is that the former is for existing camera manufacturers, and the latter is for new entry electronics manufacturers. Hypothesis 1 is supported by the confirmation of the following two points, 1 : in this analysis, the four existing camera makers are making almost the same positioning changes, 2: the transition from analog technology to integrated research and development of analog and digital technology. On the other hand, the disapproval of the establishment of Hypothesis 2 is that the positioning is different even among the new entry electronics manufacturers. Casio was included only in clusters 2 and 3, which had a high degree of technological fusion during the analysis period. On the other hand, Sony and Panasonic are in a position to support the establishment of Hypothesis 2. As the name suggests, existing camera manufacturers have been manufacturing film cameras before the digital camera market started, and are conducting R \& D for that purpose. For example, a typical standard of film used for a film camera is $35 \mathrm{~mm}$ film. The standard has been in use since the 
early 1900s and has a long history. For this reason, it is possible that the research and development positioning of high-end companies of camera manufacturers were similar. On the other hand, new entry electronics manufacturers have entered the market from digital cameras and have a short history. Therefore, it is possible that the R\&D positioning of these manufacturers was different. In addition, Sony and Panasonic may also be affected by the fact that they are manufacturers of image elements that have a low degree of technological integration among the elemental technologies of digital cameras. On the other hand, because Casio relies on the supply of element parts from other companies, it is possible that $R \& D$ was also carried out with a higher degree of technological integration that combines element technologies than individual technology elements.

\subsubsection{Consideration of Hypothesis 3}

Moreover, although Hypothesis 3 was also unsuccessful, it was found that after 2000, the positioning of the 7 companies under analysis was closer than in the 1990s. Also, from the perspective of the area such as clusters, although it is a separate area for 3 and 4, most of the clusters 2 have a high degree of analog fusion and digital ones only for the axis of dimension 1 (horizontal axis). It is located between those with a high degree of technological integration. In addition, as mentioned above, most of the products included in cluster 2 are existing camera manufacturers after 2000. In other words, it was found that the positioning of existing camera manufacturers since 2000 is located in the middle of the areas where the degree of technological integration between analog and digital is high, and is focusing on the integrated research of analog and digital. On the other hand, from the viewpoint of the axis of 1st dimension (horizontal axis), which is included in cluster 3 since the 2000s of new entry electronics manufacturers, it is located on the opposite side of cluster 2. This is because Sony, a new entry electronics manufacturer, has acquired the technology in this analysis by collaborating with Konica Minolta for the camera body mechanism and lens, which are analog technology, and by collaborating with Carl Zeiss for the lens. Panasonic has acquired technology in partnership with Leica. In this way, by acquiring analog technology through partnerships with other companies, it is possible that the result of focusing our research resources on digital technology in our field is positioning that does not approach the analog side.

\section{Summary}

In this analysis, we conducted a quantitative analysis of the research and development trends of digital cameras, in which Japanese companies account for $90 \%$ of world shipments. Japanese companies have a mix of existing camera manufacturers and new entry electronics manufacturers. The purpose of this study was to analyze and clarify patent data related to digital cameras from the viewpoint of positioning in the research and development area, regarding how the research and development of each company's specialty technology and the technology that needs to be caught up progressed. Three hypotheses were set, but only hypothesis 1 was supported. In other words, the existing camera manufacturers have moved from analog technology to integrated research and development of analog and digital technologies. Although Hypothesis 2 was not supported, Sony and Panasonic, which are new entry electronics manufacturers, moved from digital technology to integrated research and development of analog and digital technology. As set out in Hypothesis 3, after 2005, it was found that the R\&D areas of existing camera manufacturers and new entry electronics manufacturers were not close, but compared to the 
1990s. It was also found that the existing camera manufacturers are more focused on the integrated research of analog and digital compared with the new entry electronics manufacturers. The patent data of the digital camera maker is used for the analysis period of this time. If the patent data for patents filed after 2015, when commoditization progresses further, is included in the analysis target, the positioning will be closer and the establishment of Hypothesis 3 may be supported. However, patent data is usually not released until 1.5 years after application, and it takes several years to determine the data due to differences in application routes such as PCT applications. Therefore, we have a chance to make a new verification, but we would definitely like to try it. The method of this analysis seems to be effective in confirming the transition of research and development of products that combine different technologies. In addition to the digital camera field, the fields in which the fusion technology becomes a product include the electric vehicle field in which automobile technology and electronics technology, the secondary battery technology fuse, and the surgical robot field in which medical technology and robot technology fuse. Applying to these R\&D positioning is also a future issue.

\section{References}

[1] Solow, Robert M. "Technical change and the aggregate production function." The review of Economics and Statistics (1957): 312-320.

[2] Klaus Brockhoff (1989) "Forschung und Entwicklung. Planung und Kontrolle", Oldenbourg Wissenschaftsverlag

[3] Yasuda, Takehiko. "Firm growth, size, age and behavior in Japanese manufacturing." Small Business Economics 24.1 (2005): 1-15.

[4] Wernerfelt, B. (1984) "A Resource-Based View of the Firm," Strategic Management Journal, 5, 171-180.

[5] Barney, J. B. (1986) "Strategic Factor markets: Expectations, Luck, and Business Strategy", Management Science, Vol. 62, pp. 777-795.

[6] Barney, J. B. (1991) "Firm Resources and Sustained Competitive Advantage", Journal of Management, 17, 1, pp. 99-120.

[7] Prahalad, C K. and G. Hamel (1990) "The Core Competence of the Corporation," Harvard Business Review, 68, 3, 79-91.

[8] Deng Zhen, Baruch Lev, and Francis Narin, "Science and technology as predictors of stock performance," Financial Analysts Journal 55.3, 1999, pp. 20-32.

[9] Cooper Robert G., and Elko J. Kleinschmidt, "New products: what separates winners from losers?,"Journal of Product Innovation Management 4.3, 1987, pp. 169-184.

[10] Henard David H., and David M. Szymanski, "Why some new products are more successful than others," Journal of Marketing Research 38.3, 2001, pp. 362-375. 
[11] Song X. Michael, and Mark E. Parry, "A cross-national comparative study of new product development processes: Japan and the United States," Journal of Marketing 61.2, 1997, pp. $1-18$.

[12] Chery Nakata, Subin Im, Heungsoo Park, and Young-Won Had, "Antecedents and consequence of Korean and Japanese new product advantage," Journal of Business Research 59.1, 2006, pp. 28-36.

[13] Comanor William S., and Frederic M. Scherer, "Patent statistics as a measure of technical change," Journal of Political Economy 77.3, 1969, pp. 392-398.

[14] Pavitt Keith, "R\&D, patenting and innovative activities: a statistical exploration," Research Policy 11.1, 1982, pp. 33-51.

[15] Griliches, Zvi. "Patent Statistics as Economic Indicators: A Survey." Journal of Economic Literature Vol. 28, No. 4, 1990. 1661-1707.

[16] Griliches, Zvi. "Patent statistics as economic indicators: a survey." R\&D and productivity: the econometric evidence. University of Chicago Press, 1998. 287-343.

[17] Kondo Masayuki, "R\&D dynamics of creating patents in the Japanese industry," Research Policy 28.6, 1999, pp. 587-600.

[18] Artz K. W., Norman P. M., Hatfield D. E., and Cardinal L. B., "A longitudinal study of the impact of R\&D, patents, and product innovation on firm performance," Journal of Product Innovation Management 27.5, 2010, pp. 725-740.

[19] Pilkington Alan, and Romano Dyerson, "Innovation in disruptive regulatory environments: A patent study of electric vehicle technology development," European Journal of Innovation Management 9.1, 2006, pp. 79-91.

[20] Chen Yu-Shan, and Ke-Chiun Chang, "Exploring the nonlinear effects of patent citations, patent share and relative patent position on market value in the US pharmaceutical industry," Technology Analysis \& Strategic Management 22.2, 2010, pp. 153-169.

[21] Mitkova Liliana, "Marketing: a key element in patent management," International Journal of Technology Transfer and Commercialisation 4.4, 2005, pp. 487-499.

[22] Lieberman Marvin B., and David B. Montgomery, "First-mover advantages," Strategic Management Journal 9.S1, 1988, pp. 41-58.

[23] Henderson, Rebecca. "Underinvestment and incompetence as responses to radical innovation: Evidence from the photolithographic alignment equipment industry." The RAND Journal of Economics (1993): 248-270.

[24] Goto Mika, Angie Low, and Anil K. Makhija, "Diversification, Productivity, and Financial Constraints Empirical Evidence from the US Electric Utility Industry," Ohio State University, Charles A. Dice Center for Research in Financial Economics Working Paper 
Series 3, 2008 .

[25] Schnaars Steven P. Managing imitation strategies. Simon and Schuster, 2002.

[26] Bergek A., Berggren C., Magnusson T., and Hobday M., "Technological discontinuities and the challenge for incumbent firms: Destruction, disruption or creative accumulation?" Research Policy 42.6-7, 2013, pp. 1210-1224.

[27] Benner, Mary J., and Mary Tripsas. "The influence of prior industry affiliation on framing in nascent industries: The evolution of digital cameras." Strategic Management Journal 33.3 (2012): 277-302.

[28] Teece David J, "Towards an economic theory of the multiproduct firm," Journal of Economic Behavior \& Organization 3.1, 1982, pp. 39-63.

[29] Bernard Andrew B., Stephen J. Redding, and Peter K. Schott, "Multiple-product firms and product switching," American Economic Review 100.1, 2010, pp. 70-97.Comanor William S., and Frederic M. Scherer, "Patent statistics as a measure of technical change," Journal of Political Economy 77.3, 1969, pp. 392-398.

[30] Kodama, Fumio. "Technology fusion and the new R\&D." Harvard Business Review (1992): 70-78.

[31] Suzuki, Jun, and Fumio Kodama. "Technological diversity of persistent innovators in Japan: Two case studies of large Japanese firms." Research Policy 33.3 (2004): 531-549.

[32] Kodama, Fumio, and Jun Suzuki. "How Japanese companies have used scientific advances to restructure their businesses: The receiver-active national system of innovation." World Development 35.6 (2007): 976-990. 


\section{Appendix}

Table 5: Description of Analog Patent (G02B1 G02B17, G03B1 G03B19)

\begin{tabular}{|c|c|}
\hline IPC & Descriptions \\
\hline - $\mathrm{G}$ & PHYSICS \\
\hline - G02 & OPTICS \\
\hline - G02B & OPTICAL ELEMENTS, SYSTEMS, OR APPARATUS \\
\hline - G02B1/00 & $\begin{array}{l}\text { Optical elements characterised by the material of which they are made; } \\
\text { Optical coatings for optical elements }\end{array}$ \\
\hline - $\mathrm{G} 02 \mathrm{~B} 3 / 00$ & Simple or compound lenses \\
\hline - G02B5/00 & Optical elements other than lenses \\
\hline - G02B6/00 & Light guides \\
\hline - G02B7/00 & $\begin{array}{l}\text { Mountings, adjusting means, or light-tight connections, for optical } \\
\text { elements }\end{array}$ \\
\hline - G02B9/00 & $\begin{array}{l}\text { Optical objectives characterised both by the number of the components } \\
\text { and their arrangements according to their sign, i.e. }+ \text { or }-\end{array}$ \\
\hline - G02B11/00 & $\begin{array}{l}\text { Optical objectives characterised by the total number of simple and } \\
\text { compound lenses forming the objective and their arrangement }\end{array}$ \\
\hline - G02B13/00 & Optical objectives specially designed for the purposes specified below \\
\hline - G02B15/00 & Optical objectives with means for varying the magnification \\
\hline - G02B17/00 & Systems with reflecting surfaces, with or without refracting elements \\
\hline$\cdot \mathrm{G} 03$ & 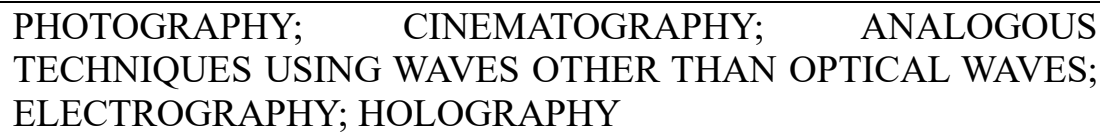 \\
\hline - G03B & $\begin{array}{l}\text { APPARATUS OR ARRANGEMENTS FOR TAKING } \\
\text { PHOTOGRAPHS OR FOR PROJECTING OR VIEWING THEM; } \\
\text { APPARATUS OR ARRANGEMENTS EMPLOYING ANALOGOUS } \\
\text { TECHNIQUES USING WAVES OTHER THAN OPTICAL WAVES; } \\
\text { ACCESSORIES THEREFOR }\end{array}$ \\
\hline - G03B1/00 & Film strip handling \\
\hline - G03B3/00 & $\begin{array}{l}\text { Focusing arrangements of general interest for cameras, projectors or } \\
\text { printers }\end{array}$ \\
\hline - G03B5/00 & $\begin{array}{l}\text { Adjustment of optical system relative to image or object surface other } \\
\text { than for focusing }\end{array}$ \\
\hline - G03B7/00 & $\begin{array}{l}\text { Control of exposure by setting shutters, diaphragms or filters, separately } \\
\text { or conjointly }\end{array}$ \\
\hline • G03B9/00 & Exposure-making shutters; Diaphragms \\
\hline - G03B11/00 & Filters or other obturators specially adapted for photographic purposes \\
\hline - G03B13/00 & $\begin{array}{l}\text { Viewfinders; Focusing aids for cameras; Means for focusing for } \\
\text { cameras; Autofocus systems for cameras }\end{array}$ \\
\hline - G03B15/00 & Special procedures for taking photographs; Apparatus therefor \\
\hline - G03B17/00 & Details of cameras or camera bodies; Accessories therefor \\
\hline - G03B19/00 & Cameras \\
\hline
\end{tabular}


Table 6: Description of Digital Patent (H04N5/22 25:33 36, H01L27/146 148)

\begin{tabular}{|c|c|}
\hline IPC & Descriptions \\
\hline$\cdot \mathrm{H}$ & ELECTRICITY \\
\hline$\cdot \mathrm{H} 04$ & ELECTRIC COMMUNICATION TECHNIQUE \\
\hline - $\mathrm{H} 04 \mathrm{~N}$ & PICTORIAL COMMUNICATION, e.g. TELEVISION \\
\hline$\cdot$ H04N5/00 & Details of television systems \\
\hline$\cdot \mathrm{H} 04 \mathrm{~N} 5 / 222$ & Studio circuitry; Studio devices; Studio equipment \\
\hline - H04N5/225 & Television cameras \\
\hline$\cdot \mathrm{H} 04 \mathrm{~N} 5 / 228$ & Circuit details for pick-up tubes \\
\hline - H04N5/232 & Devices for controlling television cameras, e.g. remote control \\
\hline - H04N5/235 & $\begin{array}{l}\text { Circuitry \{or methods\} for compensating for variation in the brightness } \\
\text { of the object }\end{array}$ \\
\hline - H04N5/238 & by influencing the optical part of the camera \\
\hline - H04N5/243 & by influencing the picture signal \\
\hline - H04N5/247 & Arrangements of television cameras \\
\hline - H04N5/253 & $\begin{array}{l}\text { Picture signal generating by scanning motion picture films or slide } \\
\text { opaques, e.g. for telecine }\end{array}$ \\
\hline$\cdot \mathrm{H} 04 \mathrm{~N} 5 / 257$ & Picture signal generators using flying-spot scanners \\
\hline$\cdot \mathrm{H} 04 \mathrm{~N} 5 / 33$ & Transforming infra-red radiation \\
\hline$\cdot \mathrm{H} 04 \mathrm{~N} 5 / 335$ & using solid-state image sensors \\
\hline$\cdot \mathrm{H} 04 \mathrm{~N} 5 / 341$ & $\begin{array}{l}\text { Extracting pixel data from an image sensor by controlling scanning } \\
\text { circuits }\end{array}$ \\
\hline - H04N5/343 & $\begin{array}{l}\text { by switching between different modes of operation using different } \\
\text { resolutions or aspect ratios }\end{array}$ \\
\hline - H04N5/345 & by partially reading an SSIS array \\
\hline - H04N5/347 & by combining or binning pixels in SSIS \\
\hline - H04N5/349 & for increasing resolution by shifting the sensor relative to the scene \\
\hline - H04N5/351 & Control of the SSIS depending on the scene \\
\hline$\cdot \mathrm{H} 04 \mathrm{~N} 5 / 353$ & Control of the integration time \\
\hline$\cdot \mathrm{H} 04 \mathrm{~N} 5 / 355$ & Control of the dynamic range \\
\hline$\cdot \mathrm{H} 04 \mathrm{~N} 5 / 357$ & Noise processing, e.g. detecting, correcting, reducing or removing noise \\
\hline - H04N5/359 & applied to excess charges produced by the exposure \\
\hline - H04N5/361 & applied to dark current \\
\hline$\cdot \mathrm{H} 04 \mathrm{~N} 5 / 363$ & applied to reset noise, e.g. KTC noise \\
\hline$\cdot \mathrm{H} 04 \mathrm{~N} 5 / 365$ & applied to fixed-pattern noise, e.g. non-uniformity of response \\
\hline - H04N5/367 & applied to defects, e.g. non-responsive pixels \\
\hline - H04N5/369 & SSIS architecture; Circuitry associated therewith \\
\hline$\cdot \mathrm{H} 01$ & BASIC ELECTRIC ELEMENTS \\
\hline - H01L & $\begin{array}{lccc}\text { SEMICONDUCTOR DEVICES; } & \text { ELECTRIC } & \text { SOLID } & \text { STATE } \\
\text { DEVICES NOT OTHERWISE PROVIDED FOR } & & \end{array}$ \\
\hline$\cdot$ H01L27/00 & $\begin{array}{l}\text { Devices consisting of a plurality of semiconductor or other solid-state } \\
\text { components formed in or on a common substrate }\end{array}$ \\
\hline - H01L27/146 & Imager structures \\
\hline - H01L27/148 & Charge coupled imagers \\
\hline
\end{tabular}

